\title{
Fabrication and characterization of monolithic piezoresistive high-g three-axis accelerometer
}

\author{
Han-II Jung, Dae-Sung Kwon and Jongbaeg Kim*
}

\begin{abstract}
We report piezoresistive high-g three-axis accelerometer with a single proof mass suspended by thin eight beams. This eight-beam design allows load-sharing at high-g preventing structural breakage, as well as the symmetric arrangement of piezoresistors. The device chip size is $1.4 \mathrm{~mm} \times 1.4 \mathrm{~mm} \times 0.51 \mathrm{~mm}$. Experimental results show that the sensitivity in $\mathrm{X}$-, Y- and Z-axes are $0.2433,0.1308$ and $0.3068 \mathrm{mV} / \mathrm{g} / \mathrm{N}$ under $5 \mathrm{~V}$ applied and the resolutions are 24.2, 29.9 and 25.4 g, respectively.
\end{abstract}

Keywords: Piezoresistive, Monolithic, Miniature, High-g, Three-axis accelerometer

\section{Background}

High-g accelerometers have been widely used in vehicle crash test, automotive air bag and shock detection applications. It is important to reduce the size of the sensor to extend the field of applications. Silicon microfabrication technique makes it possible to reduce size of the sensor as well as the production cost through batch fabrication, making it suitable for mass production. In previous researches, fabricated high-g accelerometers measure the acceleration in one-axis [1-6] or three-axis with three proof masses [7]. Compared with a three-axis accelerometer with multiple proof masses, a monolithic three-axis accelerometer is free from axis-alignment error and has advantages of smaller device size, better uniformity of the measurement signals in three sensing directions [7]. Design and analysis of monolithic three axis high-g accelerometers have been reported before [8-10], however, to our best knowledge, no actual device and experimental results were reported.

In this work, we developed a monolithic piezoresistive high-g three-axis accelerometer. The detailed description

${ }^{*}$ Correspondence: kimjb@yonsei.ac.kr

School of Mechanical Engineering, Yonsei University, 50 Yonsei-ro, Seodaemun-gu, Seoul 03722, Republic of Korea for the design, fabrication and characterization is given in the following.

\section{Design and fabrication \\ Device and circuit design}

It is desired for an accelerometer to have wide sensing range as well as the sensing capability in triaxial directions independently to be used to measure unpredictable and random-directional acceleration inputs. For this purposes, we designed mechanical structures and electrical circuits as shown in Fig. 1. Single proof mass is suspended by thin eight beams, and sixteen p-type silicon piezoresistors are formed at the top surface of the beams. We used eight beams to increase the modal frequency of the unwanted rotational mode of the proof mass, and to arrange the piezoresistors symmetrically (Fig. 1a, b). For an acceleration applied to the proof mass, resistance change caused by beam deformation (Fig. 1a) is measured as voltage difference using external circuits. One of the possible applications of the fabricated accelerometers could be shock detection in mobile devices. The small size of the accelerometer chip may allow its attachment to various components inside the mobile devices, such that we can estimate the actual acceleration values applied to each component. When a mobile device is dropped at a height of $1.5 \mathrm{~m}$, a few thousand times of the 

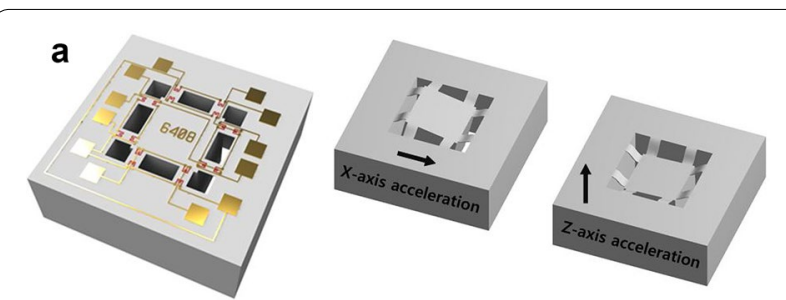

b

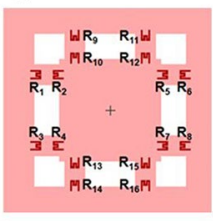

C
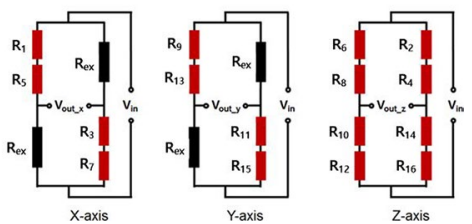

Fig. 1 a Schematic of the fabricated accelerometer and deformation under applied axial acceleration. b Arrangement of sensing piezoresistors. c Circuit diagram for three Wheatstone bridges

gravitational acceleration is applied by the impact [11]. Thus, we set the target sensing range at around 20,000 g, and the dimension of the silicon structures were designed such that the maximum stress in the beams generated by the 20,000 g axial acceleration does not exceed $1 \mathrm{GPa}$. Since the known yield stress of silicon is $7 \mathrm{GPa}$, we considered a safety factor of 7 based on our drop testing experience and considering the unexpected factors such as fabrication errors. External measurement circuits are shown in Fig. 1c. In order to detect acceleration in threedimensional space and rebuild its vector components in three-axial directions, sixteen piezoresistors and four external resistors are divided into three groups to form Wheatstone bridge circuits for detection. The resistance changes of piezoresistors for the acceleration in each of $\mathrm{x}, \mathrm{y}$ and $\mathrm{z}$ directions is shown in Table 1. When the resistance of piezoresistors are changed, $\mathrm{V}_{\text {out_x }}$ for $\mathrm{Z}$-axis bridge circuit is given by where $\mathrm{r}=\left(\mathrm{R}_{6}+\mathrm{R}_{8}\right) /\left(\mathrm{R}_{10}+\mathrm{R}_{12}\right)=\left(\mathrm{R}_{2}+\mathrm{R}_{4}\right) /\left(\mathrm{R}_{14}+\mathrm{R}_{16}\right)$ and $\eta_{A z}$ is nonlinear variable of the bridge circuit. The nonlinear term is always zero and $r=1$ from Fig. $1 \mathrm{c}$ and Table 1, when all the resistors are identical [12]. Accordingly, the simplified equations of the output signal are expressed as follows:

$$
\begin{gathered}
V_{\text {out_x }}=\frac{1}{4}\left(\frac{\Delta R_{1}+\Delta R_{5}}{R_{1}+R_{5}}+\frac{\Delta R_{3}+\Delta R_{7}}{R_{3}+R_{7}}\right) V_{\text {in }} \\
V_{\text {out_y }}=\frac{1}{4}\left(\frac{\Delta R_{9}+\Delta R_{13}}{R_{9}+R_{13}}+\frac{\Delta R_{11}+\Delta R_{15}}{R_{11}+R_{15}}\right) V_{\text {in }} \\
V_{\text {out_z }}=\frac{1}{4}\left(\frac{\Delta R_{6}+\Delta R_{8}}{R_{6}+R_{8}}-\frac{\Delta R_{2}+\Delta R_{4}}{R_{2}+R_{4}}+\frac{\Delta R_{14}+\Delta R_{16}}{R_{14}+R_{16}}\right. \\
\left.-\frac{\Delta R_{10}+\Delta R_{12}}{R_{10}+R_{12}}\right) V_{\text {in }}
\end{gathered}
$$

Acceleration in particular axis is measured by corresponding bridge circuit and the unwanted change of resistance because other axial acceleration is cancelled out. When the sensor is under $\mathrm{x}$-axial acceleration $\mathrm{A}_{\mathrm{x}}$, the same amount of compressive stress is applied to $R_{9}$ and $R_{15}$, while the same amount of tensile stress is applied to $\mathrm{R}_{11}$ and $\mathrm{R}_{13}$ due to the symmetric arrangement. Resultantly, $\Delta R_{9}=\Delta R_{15}=-\Delta R_{11}=-\Delta R_{13}$ and the change of output voltage $V_{\text {out_y }}$ becomes zero [Eq. (3)]. Similarly, $\mathrm{V}_{\text {out_x }}$ and $\mathrm{V}_{\text {out_z }}$ are measured by each bridge circuit.

\section{Fabrication}

The 3-axis accelerometer was fabricated by micromachining technique as shown in Fig. 2. A double-side polished, n-type, $\left(\begin{array}{lll}1 & 0 & 0\end{array}\right)$ oriented 4-inch silicon-on-insulator (SOI) wafer was used as a starting material. The thickness of device layer, substrate layer and buried silicon dioxide layer are 5, 500 and $1 \mu \mathrm{m}$, respectively. First, silicon

\begin{tabular}{|c|c|c|c|c|c|c|c|c|c|c|c|c|c|c|c|c|}
\hline & \multicolumn{4}{|c|}{$\mathrm{X}$-axis } & \multicolumn{4}{|l|}{ Y-axis } & \multicolumn{8}{|c|}{ Resistors of Z-axis acceleration measurement circuit } \\
\hline & $R_{1}$ & $R_{3}$ & $R_{5}$ & $R_{7}$ & $R_{9}$ & $R_{11}$ & $R_{13}$ & $R_{15}$ & $R_{2}$ & $\mathrm{R}_{4}$ & $R_{6}$ & $\mathrm{R}_{8}$ & $R_{10}$ & $R_{12}$ & $R_{14}$ & $R_{16}$ \\
\hline$A_{x}$ & $\begin{array}{l}-- \\
-\end{array}$ & $\begin{array}{l}-- \\
-\end{array}$ & $\begin{array}{l}-- \\
-\end{array}$ & $\begin{array}{l}-- \\
-\end{array}$ & - & + & + & - & +++ & +++ & +++ & +++ & + & - & - & + \\
\hline$A_{y}$ & + & - & - & + & +++ & +++ & +++ & +++ & - & + & + & - & --- & --- & --- & --- \\
\hline$A_{z}$ & ++ & ++ & -- & -- & ++ & ++ & -- & -- & -- & -- & ++ & ++ & -- & -- & ++ & ++ \\
\hline
\end{tabular}

$$
\begin{aligned}
& V_{\text {out_z }}=\frac{r}{(1+r)^{2}}\left(\frac{\Delta R_{6}+\Delta R_{8}}{R_{6}+R_{8}}-\frac{\Delta R_{2}+\Delta R_{4}}{R_{2}+R_{4}}+\frac{\Delta R_{14}+\Delta R_{16}}{R_{14}+R_{16}}-\frac{\Delta R_{10}+\Delta R_{12}}{R_{10}+R_{12}}\right) \times\left(1-\eta_{A_{z}}\right) V_{\text {in }} \\
& \eta_{A_{z}}=\left[\frac{\left(\frac{\Delta R_{6}+\Delta R_{8}}{R_{6}+R_{8}}\right)+\left(\frac{\Delta R_{2}+\Delta R_{4}}{R_{2}+R_{4}}\right)+\left(\frac{\Delta R_{14}+\Delta R_{16}}{R_{14}+R_{16}}\right)+\left(\frac{\Delta R_{10}+\Delta R_{12}}{R_{10}+R_{12}}\right)}{\left(\frac{\Delta R_{6}+\Delta R_{8}}{R_{6}+R_{8}}\right)+\left(\frac{\Delta R_{2}+\Delta R_{4}}{R_{2}+R_{4}}\right)+\left(\frac{\Delta R_{14}+\Delta R_{16}}{R_{14}+R_{16}}\right)+\left(\frac{\Delta R_{10}+\Delta R_{12}}{R_{10}+R_{12}}\right)+2}\right]
\end{aligned}
$$

\section{Table 1 Resistance changes of piezoresistors with axial acceleration}




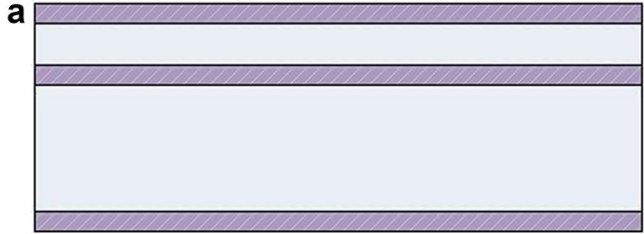

Sol wafer, oxidation

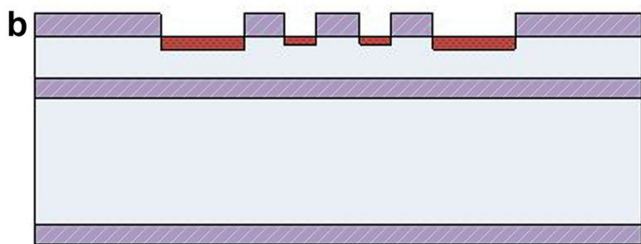

Contact pad, piezoresistor patterning, ion implantation

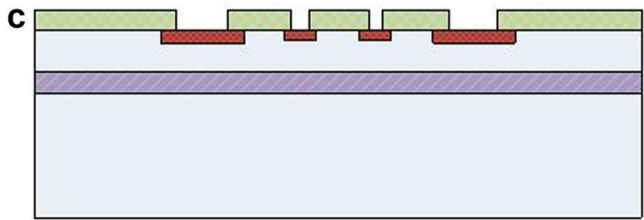

Passivation, via hole etching

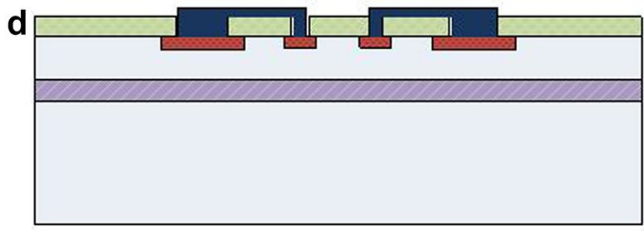

Metal line pattering e

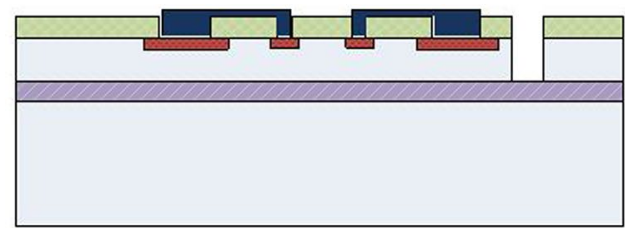

Front side patterning, DRIE

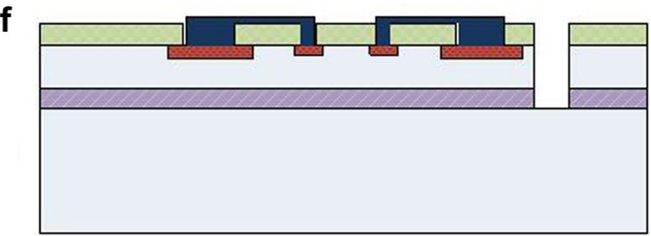

Buried oxide layer etching

g

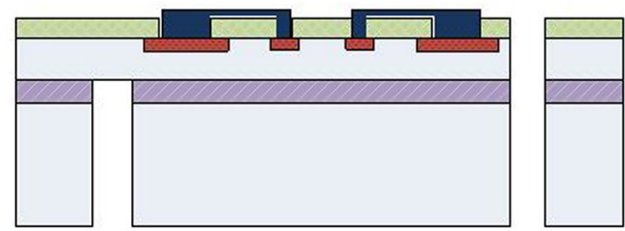

Back side patterning, DRIE

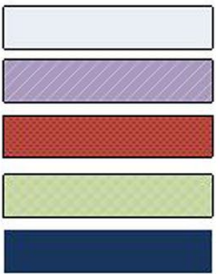

Si

$\mathrm{SiO}_{2}$

Doped region

Passivation layer

Metal line

Fig. 2 Microfabrication process flow: a $\mathrm{SiO}_{2}$ growth on $\mathrm{SOI}$ wafer. b Patterning and ion implantation to form contact pads and piezoresistors. $\mathbf{c} \mathrm{SiO}_{2}$ deposition and via hole patterning. $\mathbf{d} \mathrm{Al}$ deposition and patterning. e Patterning and sequential etching of top $\mathrm{SiO}_{2}$, device $\mathrm{Si}$ layer. $\mathbf{f} \mathrm{Buried} \mathrm{SiO}_{2}$ from the topside. $\mathbf{g}$ Patterning and dry-etching of handle Si layer from the backside

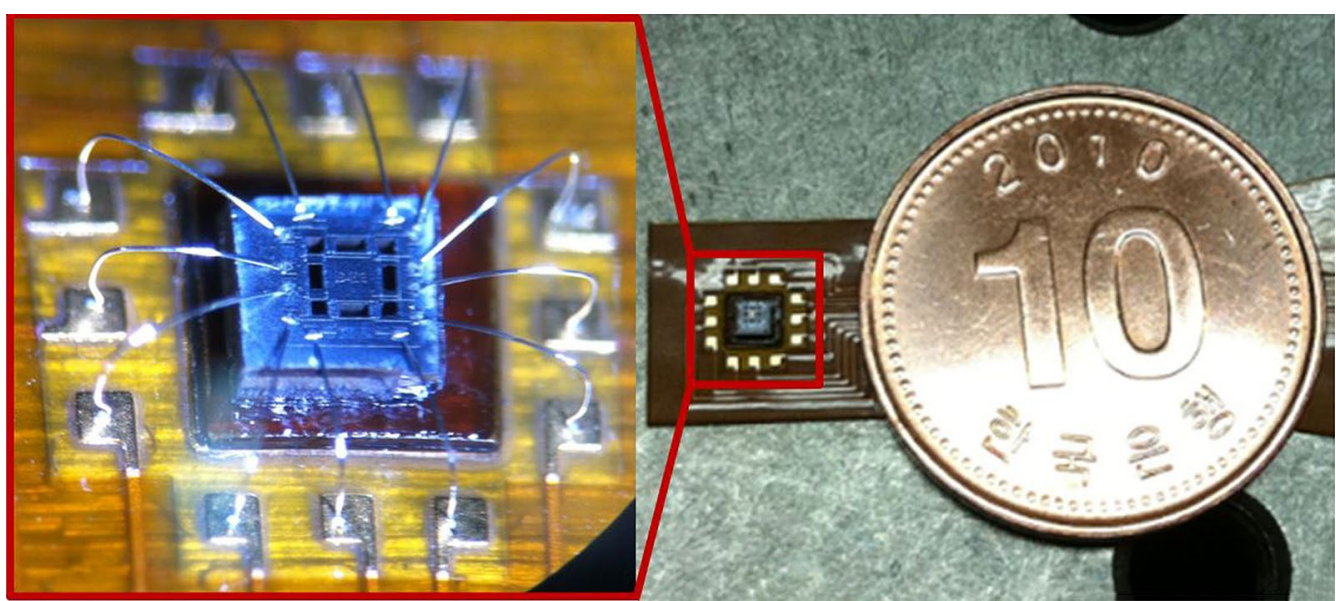

Fig. 3 Optical images of the fabricated accelerometer; An enlarged image of accelerometer attached on flexible PCB (left). Optical image of the accelerometer compared with a ten-won coin (right) 

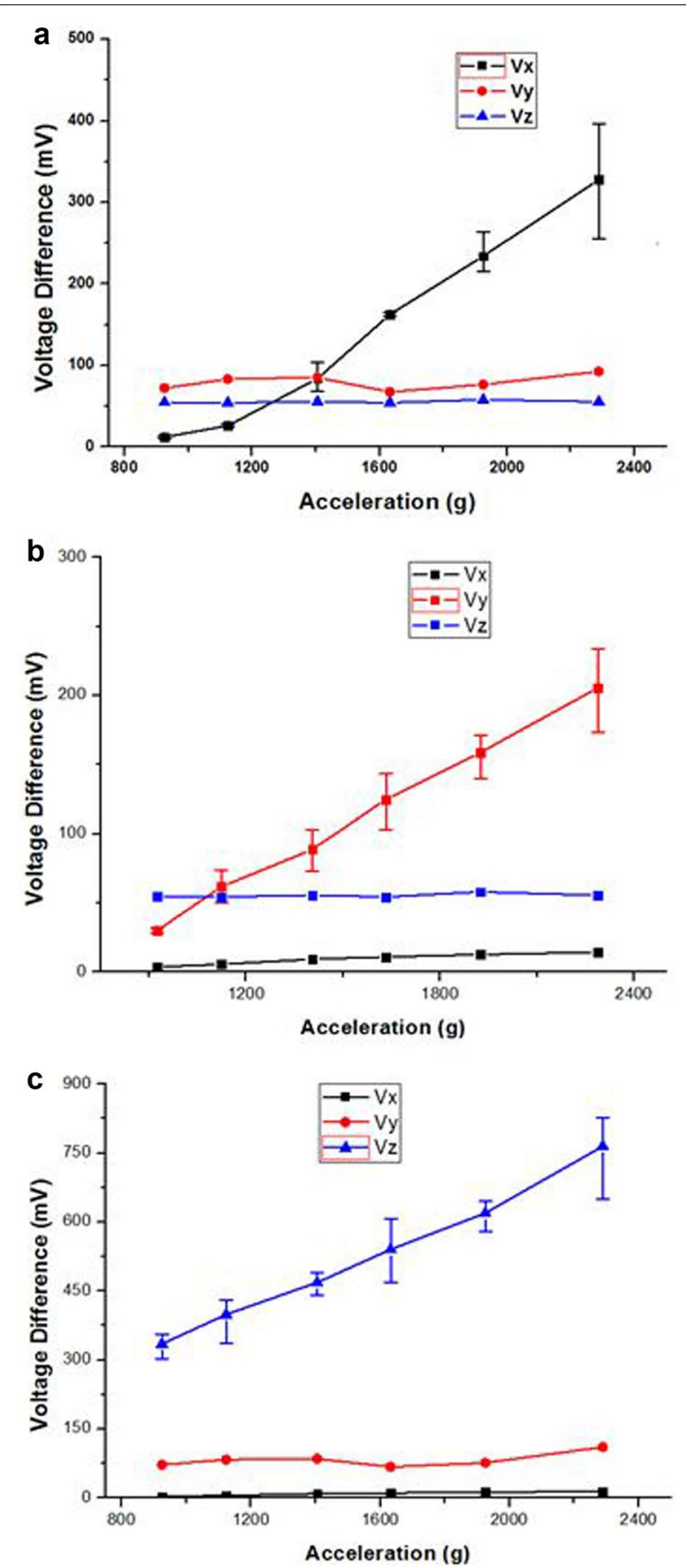

Fig. 4 Output signals as a function of axial accelerations on the $\mathbf{a} X$-, b $\mathrm{Y}$-, and $\mathbf{c} \mathrm{Z}$-axis bridge circuit

dioxide $\left(\mathrm{SiO}_{2}\right)$ layers were grown on both sides of SOI wafer by thermal oxidation (a). After the $\mathrm{SiO}_{2}$ was patterned and etched, contact pads and p-type piezoresistors were formed by implanting boron ions with the concentration of $5 \times 10^{19}$ atoms cm $\mathrm{cm}^{-3}(\mathrm{~b})$. Then $300-\mathrm{nm}$ thick $\mathrm{SiO}_{2}$ layer was deposited on the top surface as an insulating layer using plasma-enhanced chemical-vapor deposition. Etching via holes (c) for interconnection was followed by metallization to form contact pads (d). The top $\mathrm{SiO}_{2}$, device layer and buried $\mathrm{SiO}_{2}$ layer were sequentially etched to form proof mass and mechanical beams (e-f). Finally, the handle layer was deep-etched from backside to release the beams (g). It is noted that the proof mass is defined on the $500 \mu \mathrm{m}$-thick substrate layer, and is attached to the beams defined on the $5 \mu \mathrm{m}$-thick device layer.

\section{Results and discussion}

Figure 3 shows optical images of the fabricated accelerometer fixed and wire-bonded on a flexible PCB. The device chip size was $1.4 \mathrm{~mm} \times 1.4 \mathrm{~mm} \times 0.51 \mathrm{~mm}$. For calibration, both the reference accelerometer and the fabricated accelerometer were attached on a rigid metal block and this rail-guided block was dropped from various heights to apply different amount of impact to the accelerometers. The input voltage applied to the Wheatstone bridges was $5 \mathrm{~V}$ and an output signal was measured by an oscilloscope. The initial average resistance of sixteen fabricated resistors was $30.3 \mathrm{k} \Omega$. Gauge factor $(\mathrm{GF})$ of piezoresistor is defined and simplified as:

$$
G F=\frac{\frac{\Delta R}{R}}{\varepsilon}=\frac{\pi_{e f f} \sigma}{\frac{\sigma}{E}}=\frac{1}{2} \pi_{44} E
$$

where $\varepsilon, \pi_{e f f}, \pi_{44}$ and $E$ denote the strain, effective piezoresistive coefficient, shear piezoresistive coefficient $\left(85 \times 10^{-11} \mathrm{~Pa}^{-1}\right)$ and Young's modulus of silicon (163 GPa), respectively [12]. Thus the average gauge factor of the piezoresistors is calculated as 69.3.

Presented in Fig. $4 a-c$ are the test results of the fabricated accelerometer. The drop test was repeated five times and error bars represent the variation of voltage difference. Figure 4 shows that the sensor can measure each of three axes acceleration with low cross-axis sensitivity. The sensitivities for axial accelerations, $S_{x}, S_{y}, S_{z}$ on the X-, Y-, Z-axes were $0.2433,0.1308,0.3068 \mathrm{mV} / \mathrm{g} / \mathrm{V}$ and resolutions were $24.2,29.8,25.4 \mathrm{~g}$, respectively. The linearity of each directional measurement is calculated as $\mathrm{L}_{\mathrm{Ax}}=0.978, \mathrm{~L}_{\mathrm{Ay}}=0.918$ and $\mathrm{L}_{\mathrm{Az}}=0.997$ by least-square fitting. For a specific axial direction, the output signal of the corresponding resistor increased with increasing acceleration, while the others remained constant. Consequently, the fabricated accelerometer is able to detect acceleration in arbitrary direction by vector summation of all three output signals. Although there exist number of non-ideal factors including microfabrication error, misaligned attachment, and electrical noise that may cause cross-axis sensitivity, nonlinearity, and non-zero output voltage, the developed accelerometer can measure 
both the magnitude and direction of high-g acceleration in arbitrary direction.

\section{Conclusions}

A high-g three-axis accelerometer using single proof mass has been designed, fabricated, and tested. The size of the fabricated accelerometer chip is $1.4 \mathrm{~mm} \times 1.4 \mathrm{~mm} \times 0.51 \mathrm{~mm}$, and the accelerometer is suitable for impact test of portable electronic devices to evaluate mechanical robustness. To decompose acceleration in arbitrary direction into $\mathrm{x}, \mathrm{y}$ and $\mathrm{z}$ components with minimal cross-talk among each axis, we designed sixteen piezoresistors and four external resistors that construct three Wheatstone bridge circuits. The accelerometer could also be applied to detect high-g shock of projectiles or vehicles.

\section{Authors' contributions}

$\mathrm{HJ}$ and DK participated in design, fabrication, experiment and drafted the manuscript. JK conceived the study, reviewed all test methods and results, and finalized the manuscript. All authors read and approved the final manuscript.

\section{Competing interests}

The authors declare that they have no competing interests.

\section{Funding}

This work was supported by Basic Science Research Program through the National Research Foundation of Korea (NRF) funded by the Ministry of Science, ICT and future Planning (NRF-2015R1A2A1A01005496) and LG Electronics.

Received: 7 December 2016 Accepted: 31 December 2016

Published online: 10 January 2017
References

1. Wang J, Li X (2010) A high-performance dual-cantilever high-shock accelerometer single-sided micromachined in (111) silicon wafers. J Microelectromech Syst 19:1515-1520

2. Dong J, Li X, Wang Y, Lu D, Ahat S (2002) Silicon micromachined highshock accelerometers with a curved-surfaceapplication structure for over-range stop protection and free-mode-resonance depression. J Micromech Microeng 12:742-746

3. Atwell AR, Okojie RS, Kornegay KT, Roberson SL, Beliveau A (2003) Simulation, fabrication and testing of bulk micromachined $6 \mathrm{H}-\mathrm{SiC}$ high-g piezoresistive accelerometers. Sens Actuators A 104:11-18

4. Wang Z, Zong D, Lu D, Xiong B, Li X, Wang Y (2003) A silicon micromachined shock accelerometer with twin-mass-plate structure. Sens Actuators A 107:50-56

5. Fan K, Che L, Xiong B, Wang Y (2007) A silicon micromachined high-shock accelerometer with a bonded hinge structure. J Micromech Microeng 17:1206-1210

6. Eklund EJ, Shkel AM (2007) Single-mask fabrication of high-g piezoresistive accelerometers with extended temperature range. J Micromech Microeng 17:730-736

7. Dong P, Li X, Yang H, Bao H, Zhou W, Li S, Feng S (2008) High-performance monolithic triaxial piezoresistive shock accelerometer. Sens Actuators A 141:339-346

8. Lin L, Pan F, Xu J, Guo H (2010) Design of a three-axis high-g piezoresistive accelerometer. In: 5th IEEE international conference on nano/micro engineered and molecular systems, pp 773-776

9. He C, Zhang W, Xiong J, Xue C, Shi G (2012) A monolithic three-axis accelerometer with low cross-axis sensitivity. Adv Mater Res 403-408:691-696

10. Narasimhan V, Li H, Jianmin M (2015) Micromachined high-g accelerometers: a review. J Micromech Microeng 25(3):1-18

11. Tee TY, Ng HS, Lim CT, Pek E (2003) Board level drop test and simulation of TFBGA packages for telecommunication applications. In: Proceeding of 53rd electronic components and technology conference, pp 121-129

12. Amarasinghe R, Dao DV, Toriyama T, Sugiyama S (2007) Development of miniaturized 6-axis accelerometer utilizing piezoresistive sensing elements. Sens Actuators A 134:310-320

\section{Submit your manuscript to a SpringerOpen ${ }^{\odot}$ journal and benefit from:}

- Convenient online submission

- Rigorous peer review

- Immediate publication on acceptance

- Open access: articles freely available online

- High visibility within the field

- Retaining the copyright to your article

Submit your next manuscript at $\mathbf{s p r i n g e r o p e n . c o m ~}$ 